\title{
Growth-Controlled Broad Emission in Phase-Pure Two-Dimensional Hybrid Perovskite Films
}

\section{Supporting Information}

\begin{abstract}
Rhys M. Kennard, ${ }^{\dagger}$ Clayton J. Dahlman, ${ }^{\dagger}$ Juil Chung, ${ }^{\dagger}$ Benjamin L. Cotts, ${ }^{*}$ Alexander A. Mikhailovsky, ${ }^{+}$Lingling Mao, ${ }^{\dagger}$ Ryan A. DeCrescent, ${ }^{*}$ Kevin H. Stone, "Naveen R. Venkatesan, ${ }^{\dagger}$ Yahya Mohtashami, "Sepanta Assadi, "Alberto Salleo, " Jon A. Schuller, "Ram Seshadri, ${ }^{++}$and Michael L. Chabinyc. ${ }^{+*}$
\end{abstract}

† Materials Department, University of California, Santa Barbara, CA 93106, United States

‡ Department of Physics, University of California, Santa Barbara, CA 93106, United States

I| Department of Electrical and Computer Engineering, University of California, Santa Barbara, CA 93106, United States

+Department of Chemistry and Biochemistry, University of California, Santa Barbara, CA 93106, United States

- SSRL, SLAC National Accelerator Laboratory, Menlo Park, CA 94025, USA.

\# Department of Materials Science and Engineering, Stanford University, 496 Lomita Mall, Suite 102 Stanford, CA 94305, United States

*Corresponding Author: mchabinyc@engineering.ucsb.edu 


\section{Table of Contents}

\section{Methods}

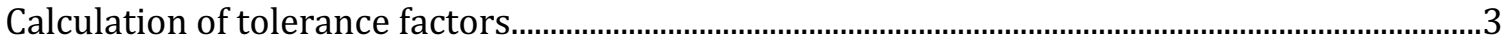

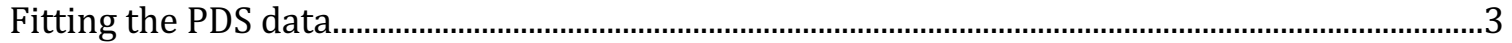

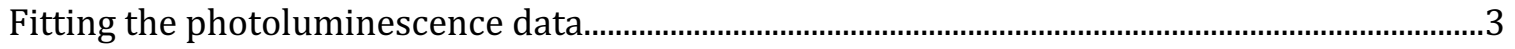

Further explanations regarding strain

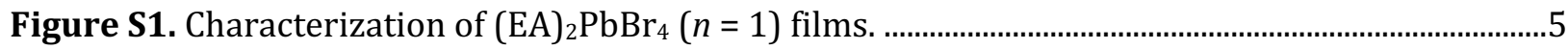

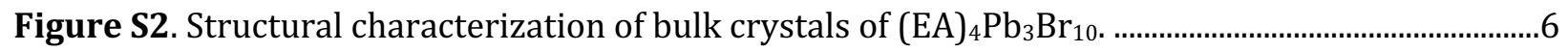

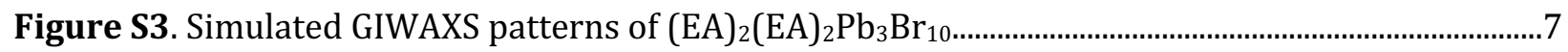

Figure S4. Near-out-of-plane (nOP) and near-in-plane (nIP) regions.......................................................

Figure S5 Fits of PL (bulk crystals, exfoliated flakes, and film) and SEM of exfoliated flakes.............10

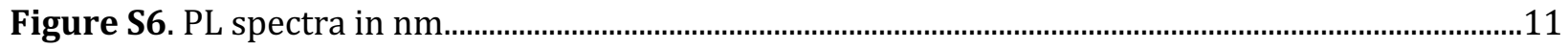

Figure S7. PL and absorbance of bulk crystals and film, with excitation $2.75 \mathrm{eV}$.................................12

Figure S8. Changes (or lack thereof) of PL with measurement conditions..............................................13

Figure S9. Excitation spectrum of the fluorimeter's lamp......................................................................14

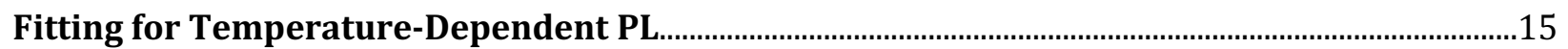

Figure S10. Fits to temperature-dependent PL from Figure 4.................................................15

Figure S11. Fits to temperature-dependent PL, assuming C is two Gaussians..........................16

Figure S12. Evolution of the fourth Gaussian from Figure S11................................................

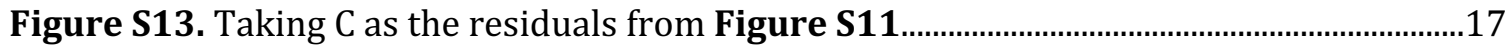

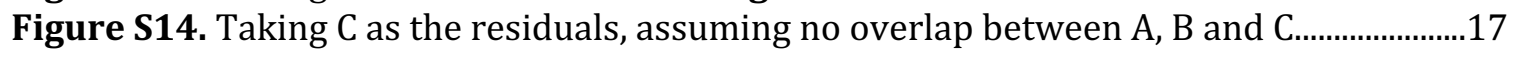

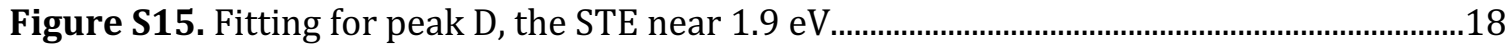

Figure S16. PDS in the 2.6-3.0 eV region with goodness of fit information..............................................19

Figure S17. SEM of the drop-cast films from Figure 6........................................................................20

Figure S18. XRD patterns of spin-cast films with different EA/Pb precursor ratio................................21

Figure S19. XRD patterns under different stability testing conditions.....................................................22

Further Discussion on 2D and 3D phases with the EA cation.................................................................24

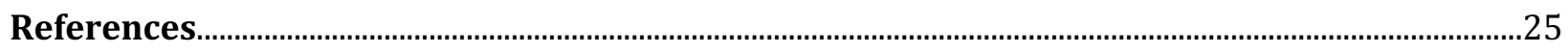




\section{Additional Methods}

\section{Calculation of Tolerance Factors}

Tolerance factors $t$ were calculated using ion sizes in 1,2 and using the equation: ${ }^{3}$

$$
t=\frac{r_{A}+r_{X}}{\sqrt{2}\left(r_{B}+r_{X}\right)}
$$

Where $r_{\mathrm{A}}, r_{\mathrm{B}}$ and $r_{\mathrm{X}}$ are the ion radii of the $\mathrm{ABX}_{3}$ structure. Table 1 below shows the ion radii used in $\mathrm{pm}$, and the resulting ts:

\begin{tabular}{|c|c|c|c|}
\hline A (EA, MA) & Pb & Br or I & $\boldsymbol{t}$ \\
\hline $274(\mathrm{EA})$ & 98 & $196(\mathrm{Br})$ & 1.13 \\
\hline $217(\mathrm{MA})$ & 98 & $196(\mathrm{Br})$ & 0.99 \\
\hline $217(\mathrm{MA})$ & 103 & $220(\mathrm{I})$ & 0.96 \\
\hline
\end{tabular}

Fitting the PDS Data (Figure 5, S13)

Following previous work, 4,5 the Urbach tails were fit according to:

$$
P D S=A \exp \left(\frac{E-E_{G}}{E_{U}}\right)
$$

Where PDS is the PDS signal, $\mathrm{A}$ is a prefactor, $E_{G}$ is the bandgap and $E_{U}$ is the Urbach energy.

\section{Fitting the Photoluminescence Data}

All peak fits were done to Gaussians using Igor Pro 8. Peaks A and B are sharp and peak D is very distinct, so A, B and D can be fit conclusively using one Gaussian per peak. However, peak C is a broad tail. It is likely that peak $\mathrm{C}$ is likely a collection of self-trapped excitons, emitting at different energies. Thus, the choice of number of Gaussians with which to fit $\mathrm{C}$ is arbitrary. To avoid over-fitting the data and drawing false conclusions, we selected the lowest-possible number of Gaussians for $\mathrm{C}$, which was often 1 , but occasionally 2 . We tried a number of ways to characterize peak $\mathrm{C}$, these are shown in Figures S10-S14 (see: “Fitting for Temperature-Dependent PL" page 15). We also attempted various ways to fit the data, including fixing the energy values, but leaving the energies as free variables consistently produced the best fits. Further work is needed to fully understand the nature of $\mathrm{C}$, which we find to be either an STE or a phonon replica.

\section{Further Explanations regarding Strain}

Prior work on 1-dimensional $\mathrm{Pb}-\mathrm{Br}$ compounds found that the broad emission intensity sharply decreased with respect to the free exciton emission when $0.5 \mathrm{GPa}$ of pressure (or stress) was applied. 
6 The proposed mechanism for this was that under stress, the bond lengths and angles become closer to those of the ideal cubic $\mathrm{MAPbB}_{3} .{ }^{6}$ While the directional Young's modulus of $(\mathrm{EA})_{2}(\mathrm{EA})_{2} \mathrm{~Pb}_{3} \mathrm{Br} \mathrm{r}_{10}$ is not known, we can estimate that it is between the values for $(\mathrm{BA})_{2}(\mathrm{MA})_{2} \mathrm{~Pb}_{3} \mathrm{Br}_{10}$ and $\mathrm{MAPbBr}_{3}$, or 4 $\mathrm{GPa}<E<30 \mathrm{GPa}$. ${ }^{7,8}$ In terms of strain, these stress values correspond to $12.5 \%>\varepsilon>1.7 \%$, using the simple stress-strain relationship $\sigma=E \varepsilon$. Although these values represent a broad, range, it is reasonable that the strain values measured in Figure 2 could be big enough to cause emission changes. 
(a)

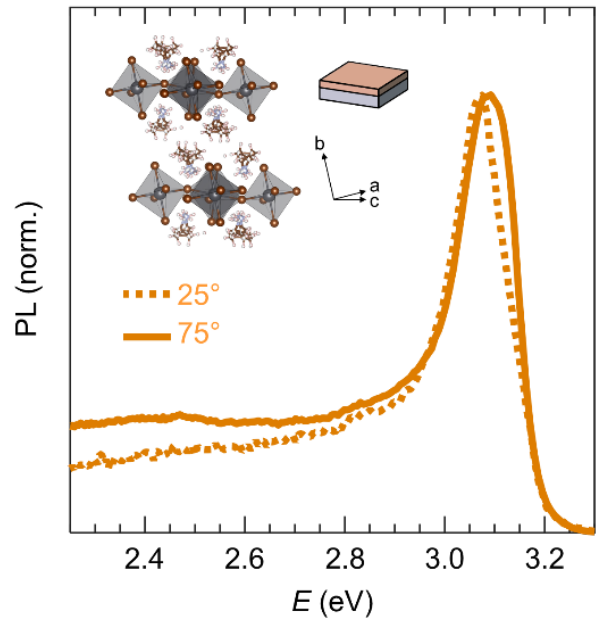

(c)

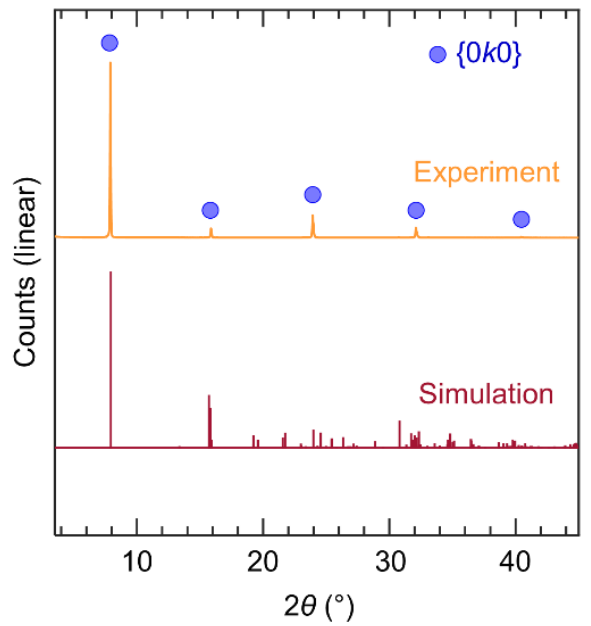

(b)

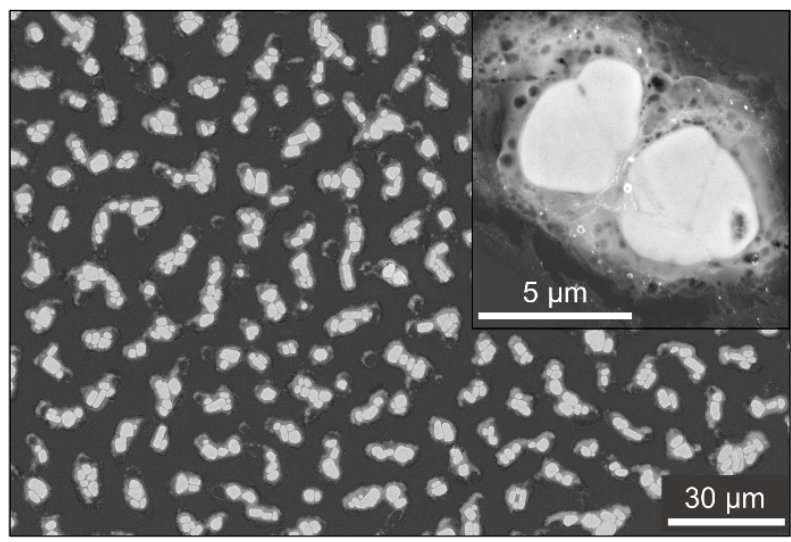

(d)

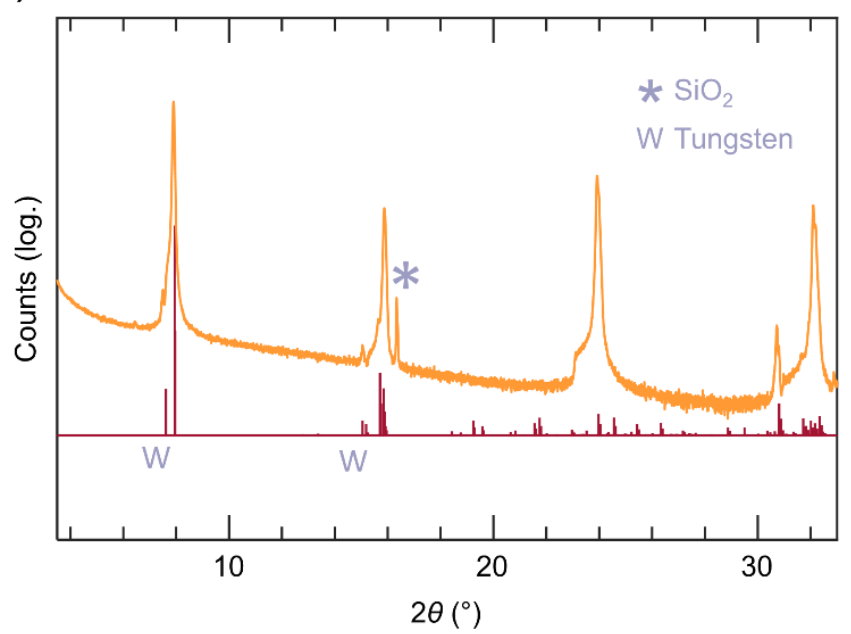

Figure S1. Characterization of $(\mathrm{EA})_{2} \mathrm{PbBr}_{4}(n=1)$ films. (a) Photoluminescence emission (3.94 eV excitation) at 25- and 75-degree collection angles. (b) Scanning Electron Microscopy of an $n=1$ film. XRD patterns plotted on (c) linear and (d) log scales. A simulated pattern of (EA) ${ }_{2} \mathrm{PbBr}_{4}$ assuming no preferred crystallite orientation, and based on the published structure, is shown as well. The copper $\mathrm{X}$-Ray source has tungsten contamination (1.4764 $\AA$ ), resulting in occasional extra peaks. The simulation in (d) shows the extra peaks from Tungsten-X-Rays as well.

The films appear to be (EA) ${ }_{2} \mathrm{PbBr}_{4}$ from X-Ray and photoluminescence characterizations. The crystallites seem to orient with the long $b$ axis normal to the substrate, as evidenced by the strong diffraction from $(0 k 0)$ peaks. The photoluminescence emission energies match well those of published crystals. ${ }^{9}$ However, as with $(\mathrm{EA})_{2}(\mathrm{EA})_{2} \mathrm{~Pb}_{3} \mathrm{Br}_{10}$, the broad emission is considerably weakened in favor of the free exciton emission. The large size of $(\mathrm{EA})_{2} \mathrm{PbBr}_{4}$ suggests that grain size is not the predominant contributor to this emission change. Other potential factors (e.g., growth kinetics, strain) are discussed in the main text. 

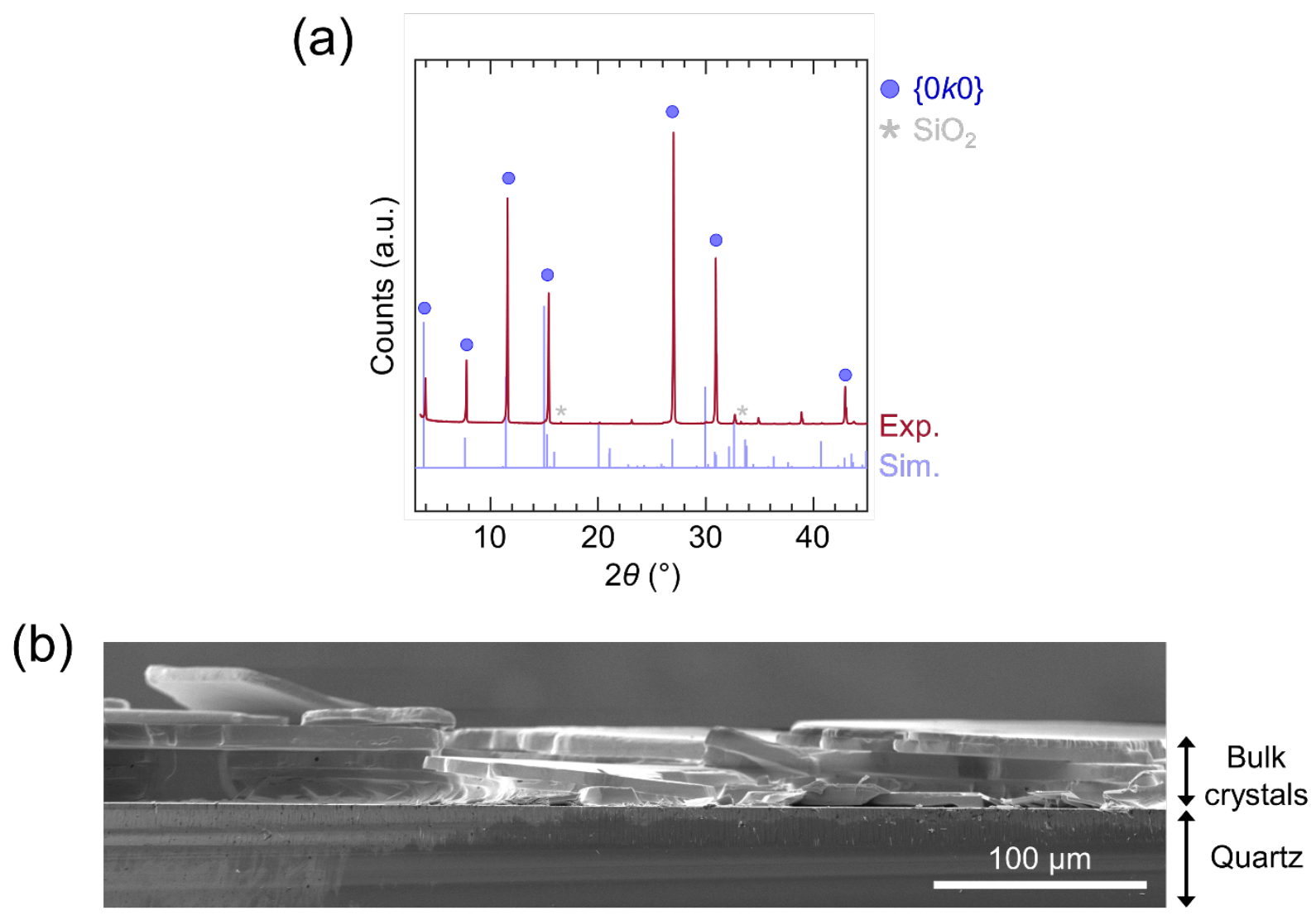

Figure S2. Structural characterization of bulk crystals of $(\mathrm{EA})_{4} \mathrm{~Pb}_{3} \mathrm{Br}_{10}$. (a) Experimental XRD pattern of bulk crystals of $(\mathrm{EA})_{2}(\mathrm{EA})_{2} \mathrm{~Pb}_{3} \mathrm{Br}_{10}$. A simulated (Sim.) powder pattern is also shown below (calculated assuming no preferred crystallite orientation - see Experimental Section). The crystals were dropped onto quartz substrates, and seem to preferentially orient with $(0 k 0)$ parallel to the substrate, given the predominance of $(0 \mathrm{k} 0)$ observed in the pattern. Quartz peaks are indicated with an asterisk. (b) Cross-sectional SEM of the bulk crystals, revealing thickness $>10$ microns. 


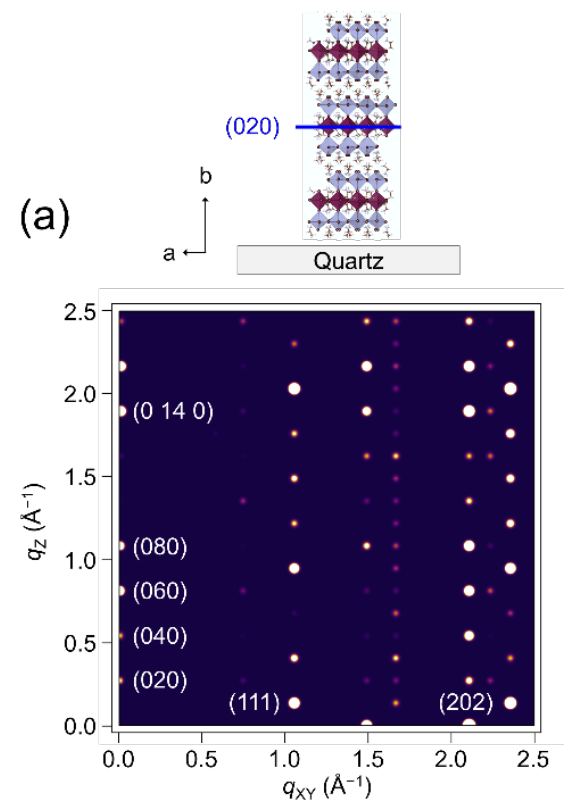

(c)

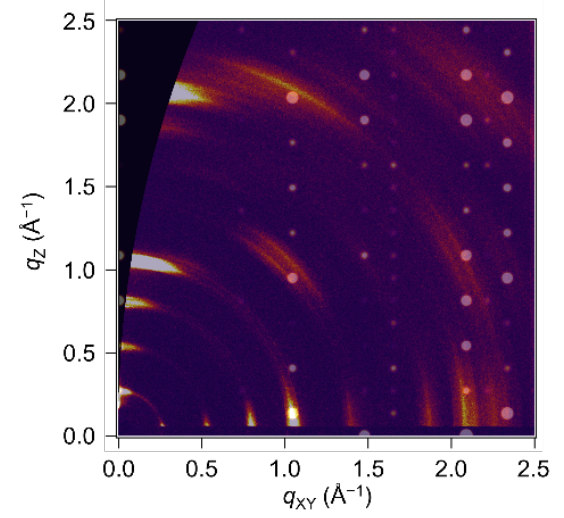

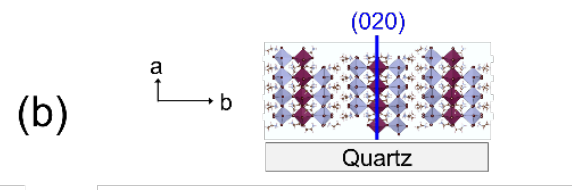

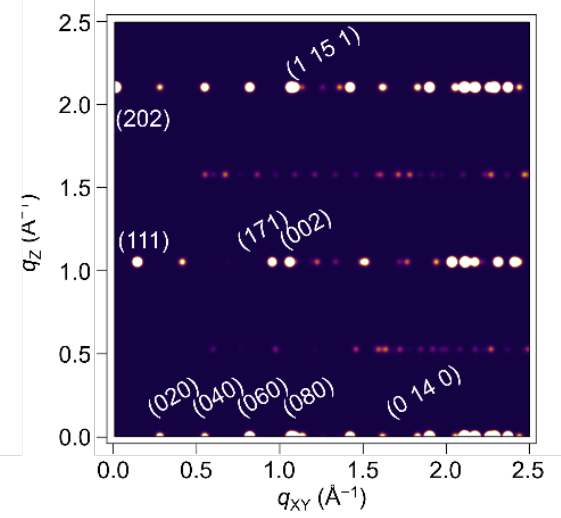

(d)

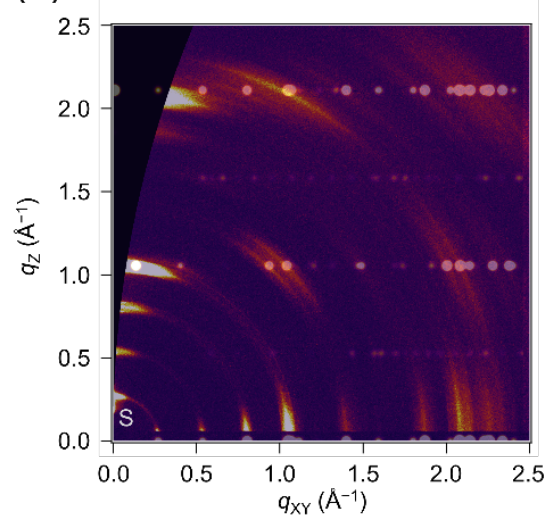

(e)

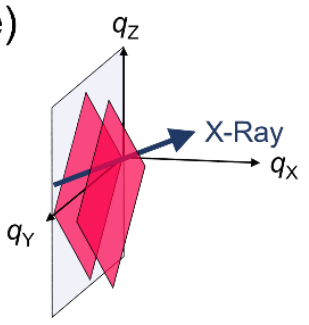

(f)

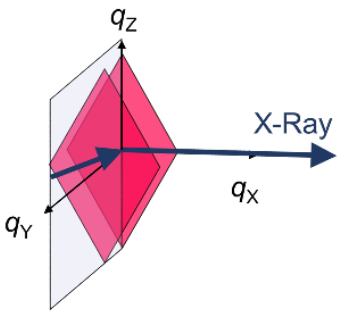

(g)

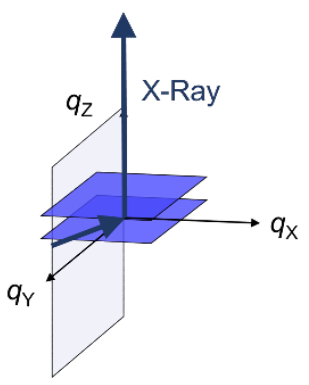

Figure S3. Simulated GIWAXS pattern of (EA) $)_{2}(\mathrm{EA})_{2} \mathrm{~Pb}_{3} \mathrm{Br}_{10}$ assuming (a) full out-of-plane crystallite orientation and (b) full in-plane crystallite orientation. Out-of-plane- and in-plane-oriented crystallites are shown above the simulated pattern, with the (020) plane for reference. Main peaks are indexed. (c)-(d) Overlay of these simulated patterns with the experimental (EA) ${ }_{2}(E A)_{2} \mathrm{~Pb}_{3} \mathrm{Br}_{10}$ GIWAXS film pattern from Figure 2, with main peaks indexed. S indicates spillover from the undiffracted beam. (e)-(g) Diffraction or lack thereof for planes (e) aligned along $q_{\mathrm{Y}}$ (lack of diffraction), (f) aligned along $q_{\mathrm{x}}$ (diffraction) and (g) aligned along $q_{\mathrm{Z}}$ (diffraction). Diffraction peaks in $q_{\mathrm{XY}}$ are less intense than those in $q_{\mathrm{Z}}$ because a smaller fraction of planes in $q_{\mathrm{XY}}$ are probed; whereas all planes in $q_{\mathrm{z}}$ are probed.

The main peaks of the experimental pattern matched with those of the simulated patterns. Simulated patterns were made using the CIF file from prior work and Python code written in house. 10,11 The (0k0), (111) and (202) peaks in the experimental pattern matched well with their expected locations in-plane and out-of-plane (Figure S3c-d). This agreement confirmed the presence of the two preferred crystallite orientations. In order to assign off-axis peaks (i.e., not along $q_{\mathrm{Z}}$, or $q_{\mathrm{XY}}$ ), we overlaid the simulated patterns for crystallites with out-of-plane and in-plane orientation with the experimental GIWAXS pattern of Figure 2. Particularly good agreement for off-axis peaks was found 
when the crystallites have in-plane orientation (Figure S3d), suggesting a greater proportion of inplane-oriented crystallites. Geometric factors, explained in the caption and in Figure S3e-g, cause greater probing of peaks in $q_{\mathrm{Z}}$ than in $q_{\mathrm{XY}}$, which likely explains why the $(0 k 0)$ peaks along $q_{\mathrm{Z}}$ appear more intense than those along $q_{\mathrm{XY}}$. In addition, due to the similar location of different peaks (especially near $q_{\mathrm{Z}}, q_{\mathrm{XY}}=1$ or 2 ), some peaks may have several possible assignments. Some of the higher-order peaks (i.e., peaks near $q_{\mathrm{Z}}$ and $q_{\mathrm{XY}}=2.5$ ) were weaker in the experimental pattern than in the simulations. This is due to two reasons: 1) the film is both thin and disordered (strain, two crystallite orientations), so higher-order peaks are weakened and 2) the simulation took only structure factors into account, and no other factors such as Debye-Waller, which make higher-order peaks weaker. Overall, the experimental GIWAXS pattern matched well the simulated GIWAXS patterns, particularly for in-plane crystallite orientation. 
(a)

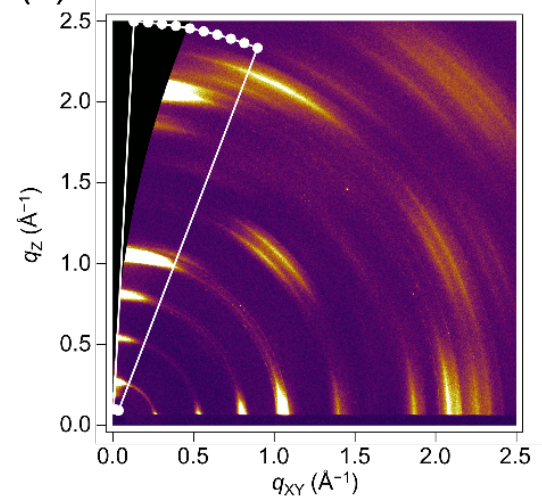

(b)

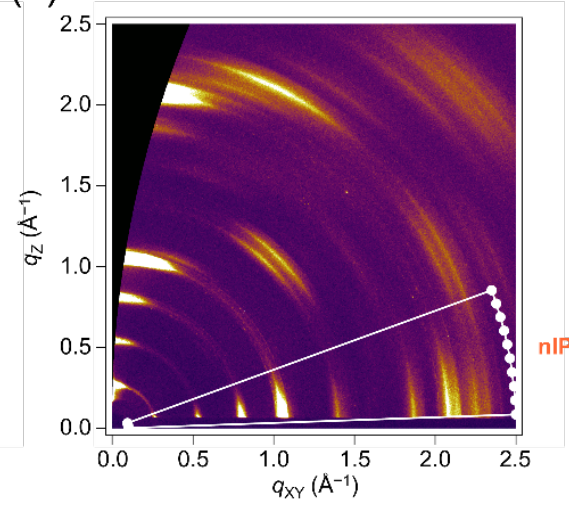

(c)

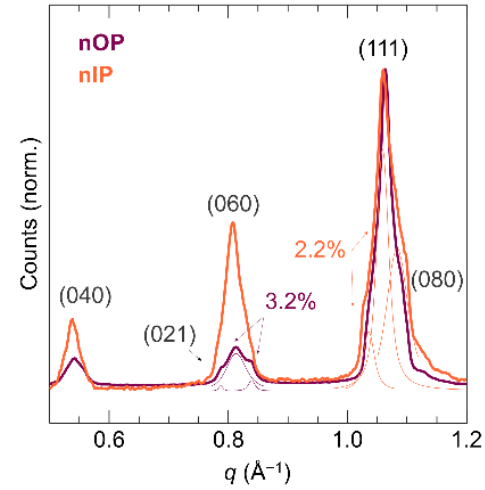

Figure S4. (a) Near-out-of-plane (nOP) and (b) near-in-plane (nIP) regions used to obtain the patterns in Figure $2 \mathbf{d}$. The nOP region covers angles between $3^{\circ}$ and $21^{\circ}$ and the nIP region covers angles between $70^{\circ}$ and $88^{\circ}$. Both sections were chosen to have equal angular spread of $18^{\circ}$. (c) Integrated and normalized nOP and nIP patterns, with relative \% strain of select peaks. Peak fits are also shown in the thinner lines. 

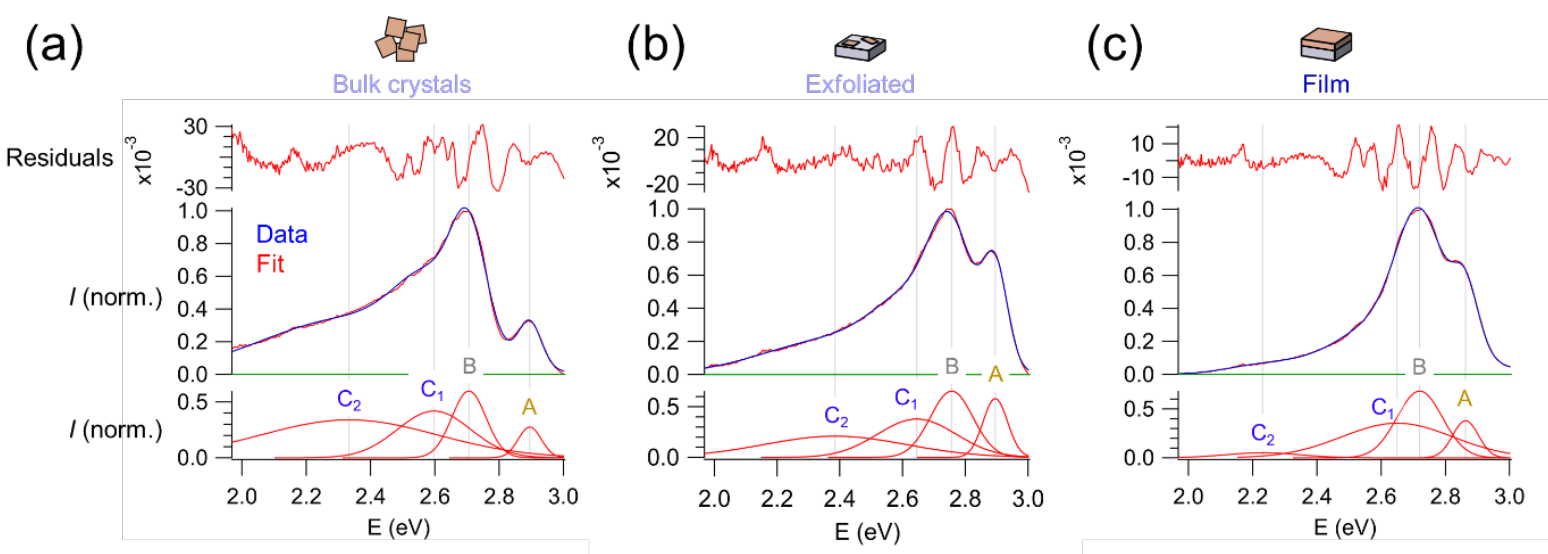

(d)

(e) Gaussian Area

(f)
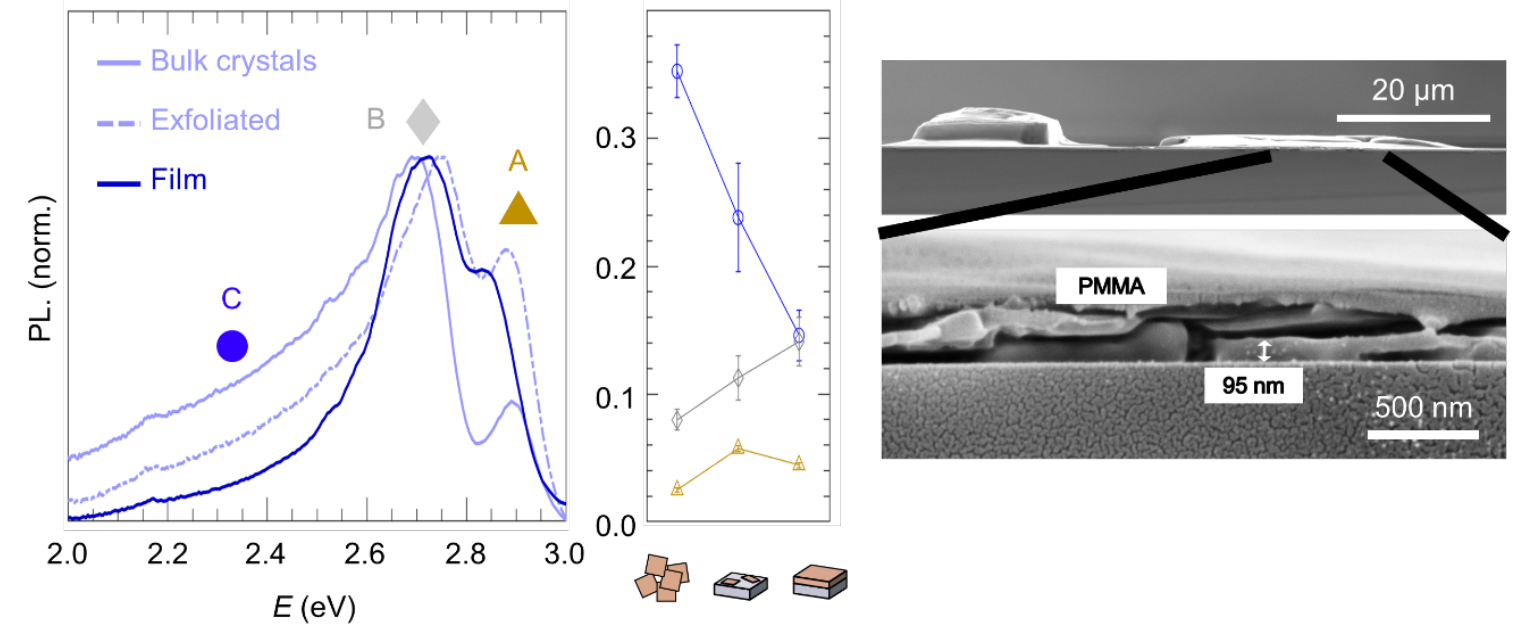

Figure S5 (a-c) Fitting of the photoluminescence emission of (a) bulk crystals, (b) exfoliated flakes, (c) film of (EA) ${ }_{4} \mathrm{~Pb}_{3} \mathrm{Br}_{10}$ (see Figure 3 for PL from the main text from fluorimeter). All spectra were fit to multiple Gaussians using Igor Pro 8. All measurements were performed with an excitation wavelength of $370 \mathrm{~nm}\left(3.35 \mathrm{eV}\right.$ - see Experimental Section) and an angle of $75^{\circ}$. (d) Overlay of the PL of crystals, flakes and film. (e) Gaussian area of peaks A, B, and C, where the area for C is the sum of the areas of $\mathrm{C}_{1}$ and $\mathrm{C}_{2}$. (f) Cross-sectional SEM of the exfoliated flakes on quartz, with a PMMA capping layer. The flake thickness was highly variable. The origins of differences in emission are discussed in the main text.

To examine how exfoliation may change emission from $\mathrm{B}$ and $\mathrm{C}$, we exfoliated bulk crystals of (EA) ${ }_{4} \mathrm{~Pb}_{3} \mathrm{Br}_{10}$, and obtained a variety of thicknesses, with some as low as $95 \mathrm{~nm}$. Exfoliated flakes of (EA) ${ }_{4} \mathrm{~Pb}_{3} \mathrm{Br}_{10}$ were prepared from bulk crystals via the standard scotch-tape method. 12,13 Fits for peaks $\mathrm{A}$ and $\mathrm{B}$ are fairly certain, as $\mathrm{A}$ and $\mathrm{B}$ are narrow peaks. However, $\mathrm{C}$ is a broad tail, and necessitated being decoupled into several peaks, $C_{1}$ and $C_{2}$. Different fits were tried; in all cases the total area of $\mathrm{C}$ goes as bulk crystals $>$ exfoliated flakes $>$ film. It is clear that the proportions of $B$ and $C$ both change with sample preparation. Exfoliation is not completely decoupled from strain, as mechanical exfoliation involves applying pressure to separate the sheets, and this may change the cation stacking. 
(a)

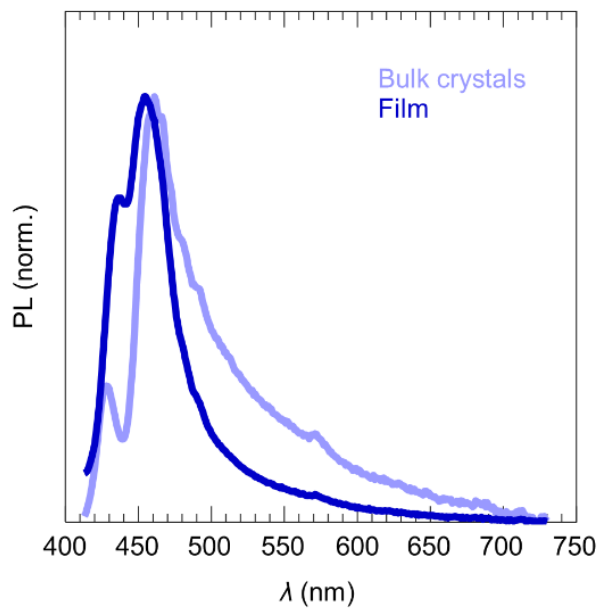

(b)

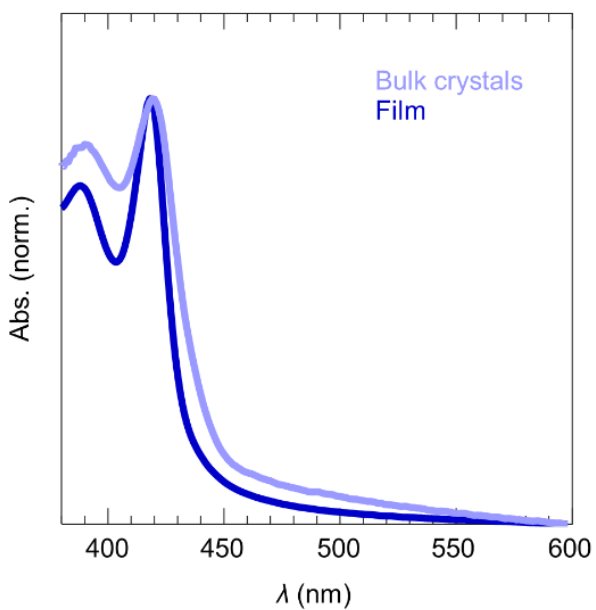

Figure S6. (a) Photoluminescence emission and (b) absorbance of spin-cast films and powders of $(\mathrm{EA})_{2}(\mathrm{EA})_{2} \mathrm{~Pb}_{3} \mathrm{Br}_{10}$ from Figure 3, shown here in $\mathrm{nm}$ (excitaiton $3.35 \mathrm{eV} ; 370 \mathrm{~nm}$ ).

The optical data were collected in $\mathrm{nm}$ and converted to eV. For emission spectra, a Jacobian transformation ${ }^{14}$ was applied to the intensities:

$$
\text { Intensity }_{\text {Converted }}=\frac{1240 \times \text { Intensity }_{\text {Collected }}}{\text { Energy }^{2}}
$$

Where Intensity Converted $_{\text {is }}$ is the PL intensity of the plot in $\mathrm{eV}$, Intensity Collected $_{\text {is }}$ the PL intensity of the plot in nm, and Energy is 1240/wavelength. 


\section{Excitation at $2.75 \mathrm{eV}$}

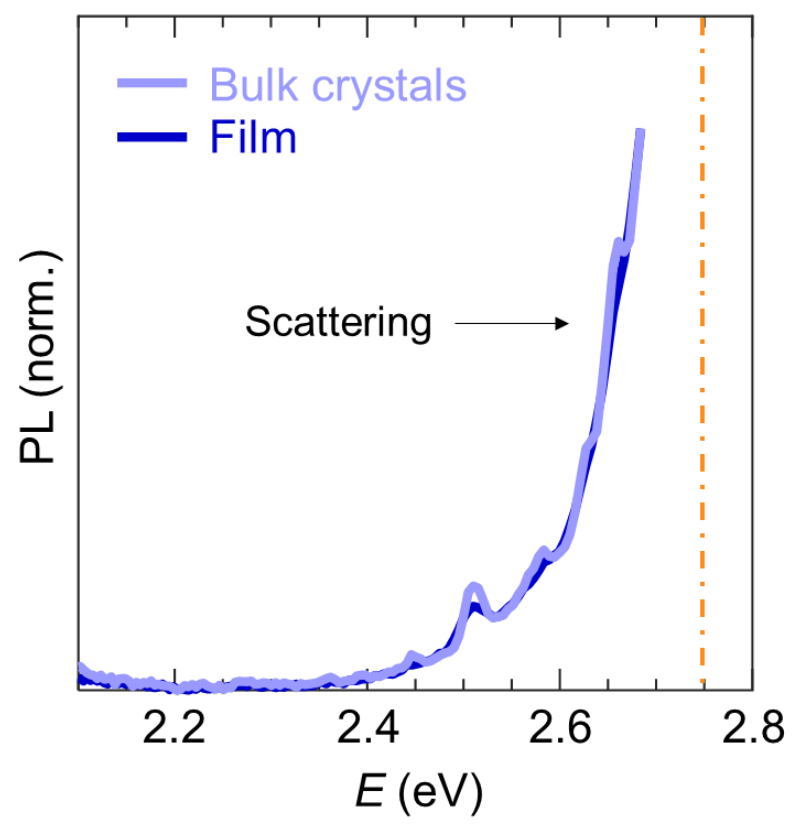

Figure S7. Photoluminescence emission (75-degree collection angle) of $(\mathrm{EA})_{2}(\mathrm{EA})_{2} \mathrm{~Pb}_{3} \mathrm{Br}_{10}$ bulk crystals and film. The excitation was selected to be $2.75 \mathrm{eV}$ to verify that broad emission only occurs when exciting the material above the absorption edge. Despite use of a long-pass filter, some scattering was observed in all samples characterized in this study at energies closest to the excitation energy, most noticeably when no (or little) photoluminescence was present in the sample. Here, the only signal observed was this scattering, confirming that sub-onset excitation does not produce broad emission. 

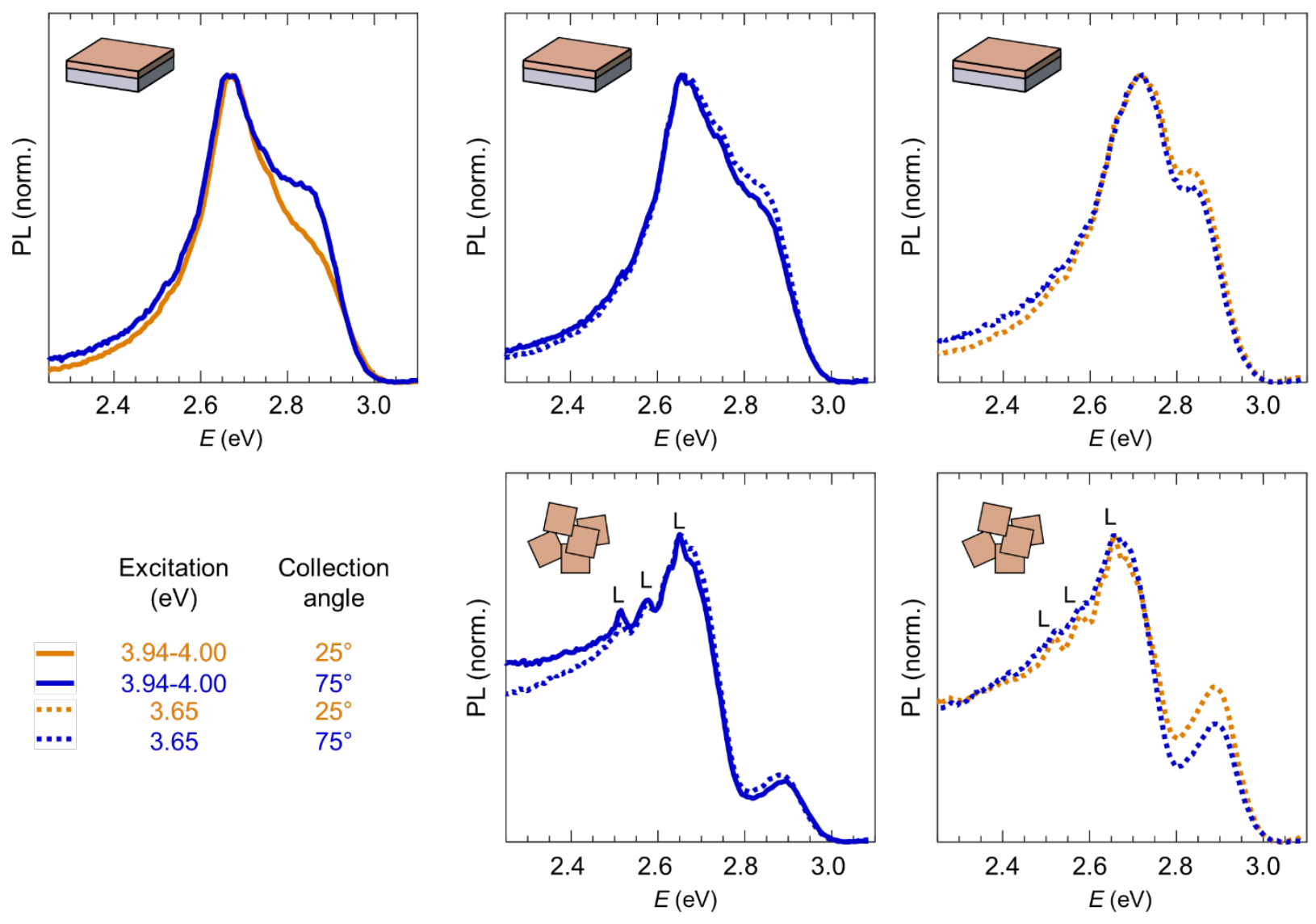

Figure S8. Changes (or lack thereof) in emission in films and bulk crystals of $(\mathrm{EA})_{2}(\mathrm{EA})_{2} \mathrm{~Pb}_{3} \mathrm{Br}_{10}$ with excitation energy and/or collection angle. Although some variations in the free exciton: broad emission proportions were observed when changing angle and/or excitation, the films consistently had a greater proportion of free exciton emission, and the bulk crystals consistently had a greater proportion of broad emission. "L" stands for scattered light from the excitation lamp (see Figure S9). 


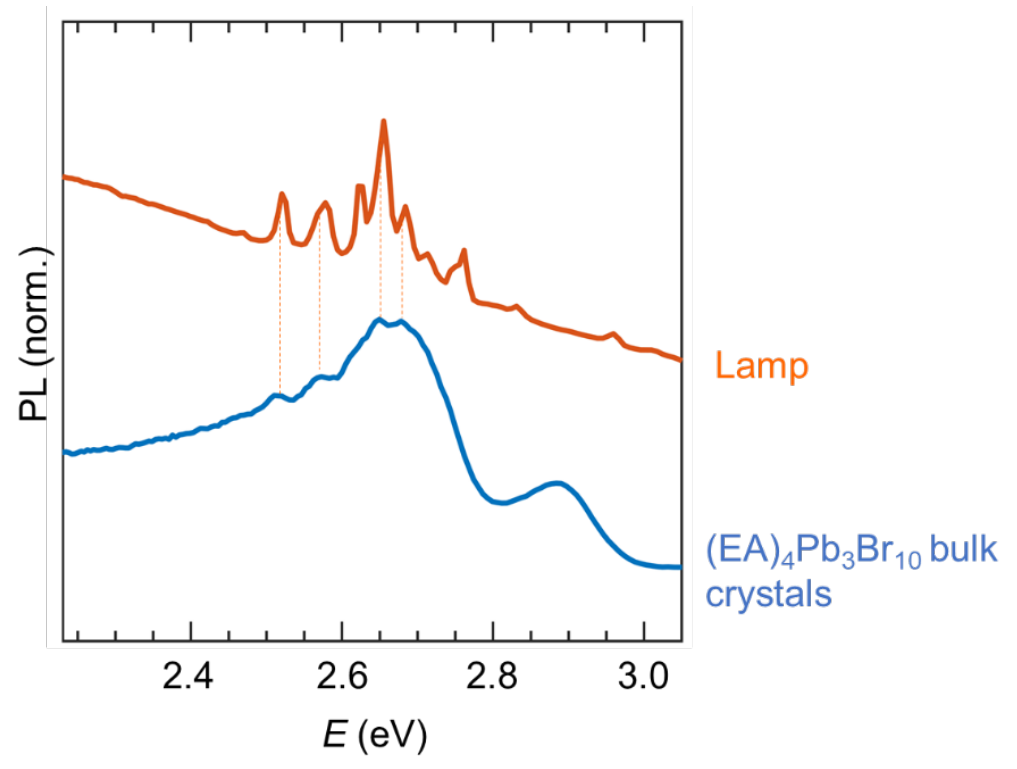

Figure S9. Excitation spectrum of the lamp, and photoluminescence emission of $(\mathrm{EA})_{4} \mathrm{~Pb}_{4} \mathrm{Br}_{10}$ bulk crystals from Figure 3. Some small peaks appear in the bulk crystal samples, due to incomplete filtering of the lamp by the fluorimeter's monochromator. When spectra are collected using a laser and CCD detector (Figure 4) instead of a monochromator-based fluorimeter (Figure 3), the sharp peaks disappear, but the proportions of $\mathrm{A}, \mathrm{B}$ and $\mathrm{C}$ remain similar. This confirms that the proportions of $\mathrm{A}, \mathrm{B}$ and $\mathrm{C}$ are inherent to the materials studied in both bulk crystals and films, and that the small, sharp peaks are from the fluorimeter's lamp. 


\section{Fitting for Temperature-Dependent PL}
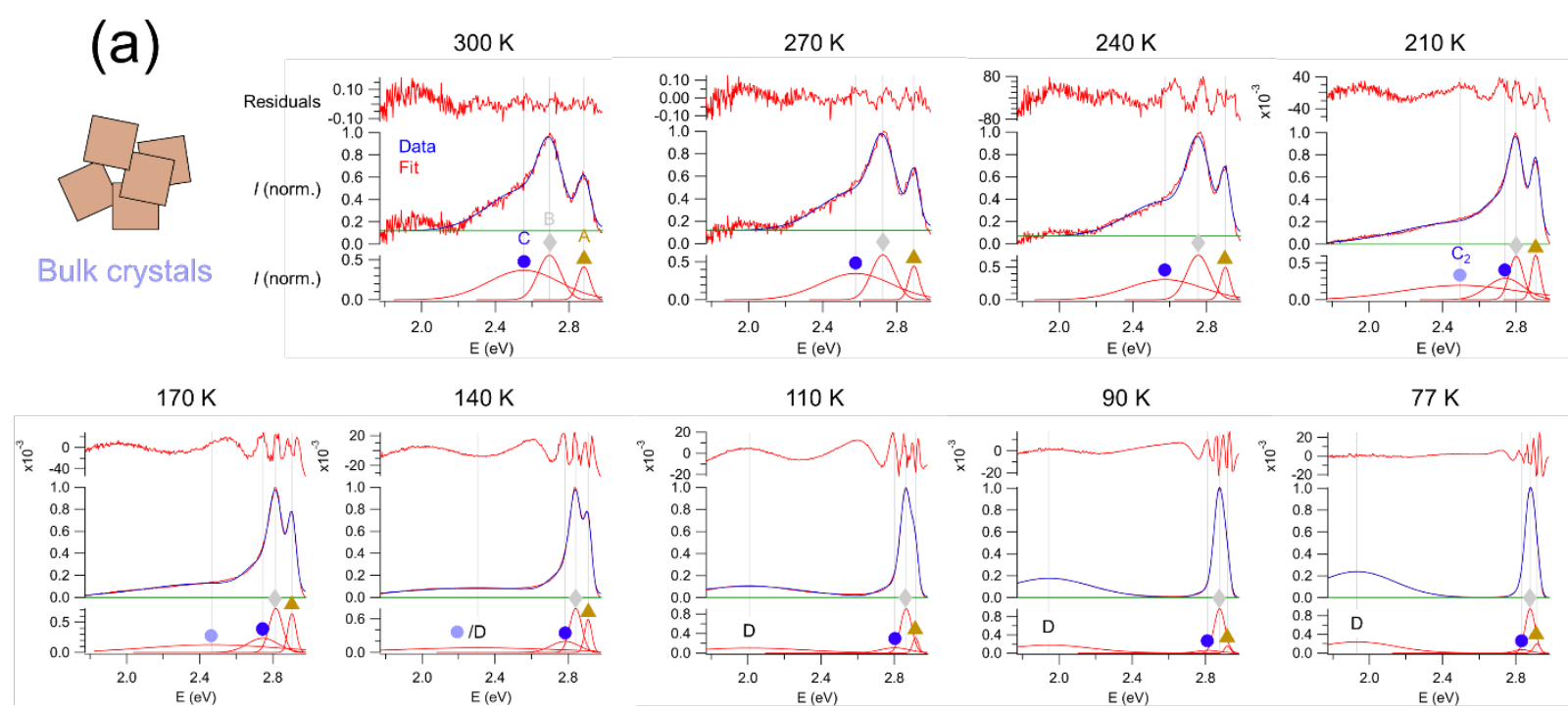

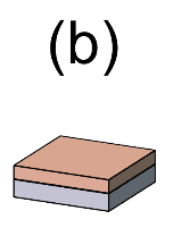

Film
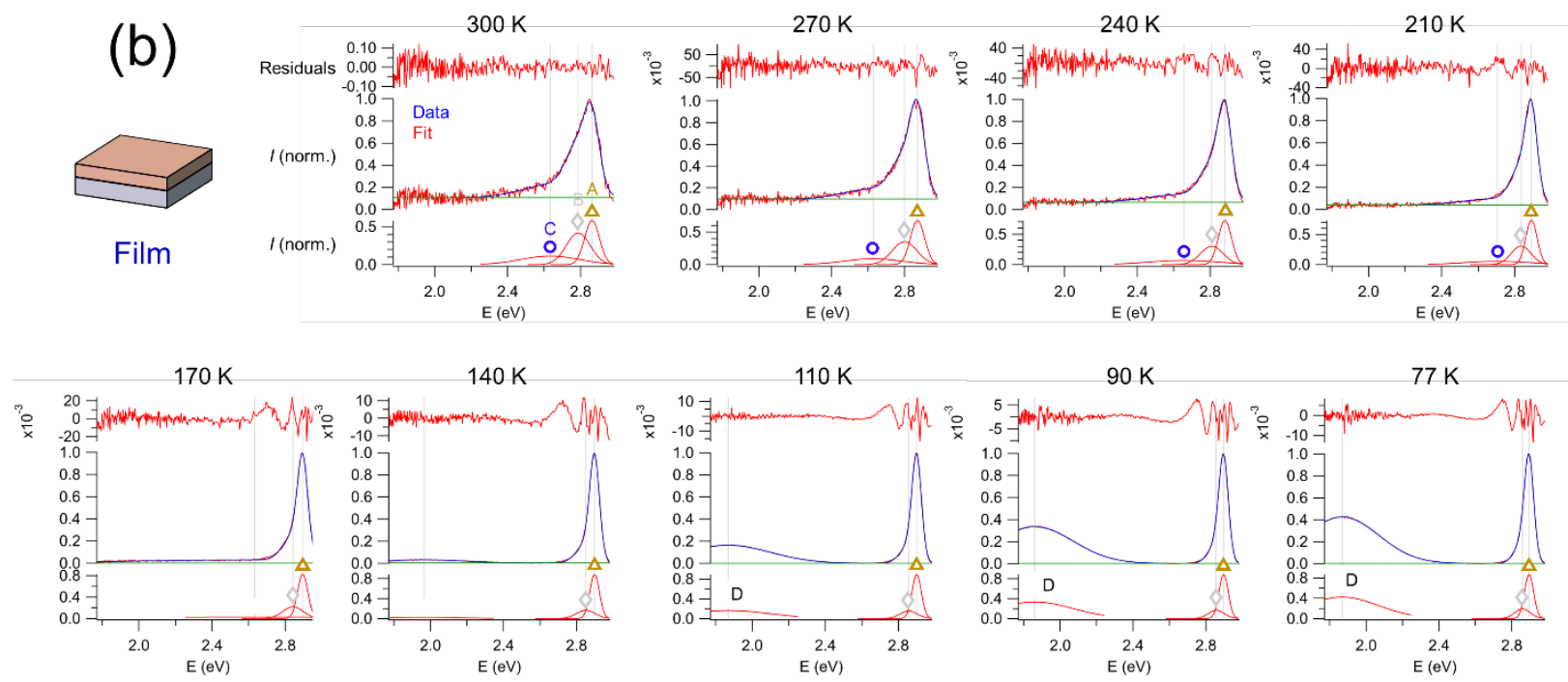

Figure S10. Temperature-dependent photoluminescence emission of the (a) bulk crystals and (b) film of $(\mathrm{EA})_{4} \mathrm{~Pb} \mathrm{~b}_{3} \mathrm{Br} 10(\mathrm{see}$ Figure 4). All spectra were fit to multiple Gaussians using Igor Pro 8. Measurements were performed with an excitation wavelength of $370 \mathrm{~nm}(3.35 \mathrm{eV})$, using a pulsed laser, and at an angle of $90^{\circ}$ (see Experimental Section). Differences between these fits and those from Figure $\mathbf{5} 5$ may be due to different experimental conditions (notably angle), as lowerenergy features can sometimes vary in intensity with angle (Figure S8). ${ }^{12,13}$ However, all trends remain the same.

\section{Methodology for fitting and determining trends}

The main challenge with fitting these spectra is that $C$ is a broad tail. Therefore, while peaks A (free exciton), B (phonon-coupled) and D (STE) are fairly obvious, it is not clear how many peaks should be used to fit C. Depending on the instrument setup, it was found that 2 Gaussians were needed to fit $\mathrm{C}$ at $300 \mathrm{~K}$ (Figure 3, S5) or only one (Figure S10). In the main text (Figure 4), we reported results 
using the lowest possible number of Gaussians, so as to not overfit the data. Below, we show additional fits, to confirm that $\mathrm{C}$ disappears and blue-shifts with cooling.

We tried to keep the position of C constant in Figure S10; this resulted in unsuccessful fits (the fit curve was not touching the experimental spectrum), providing indication that $\mathrm{C}$ likely blue-shifts.

We then tried to always use two Gaussians used for C, in order to match Figure S5. A clear blue-shift is seen for $\mathrm{C}_{1}$; trends for $\mathrm{C}_{2}$ are less clear (Figure S11):
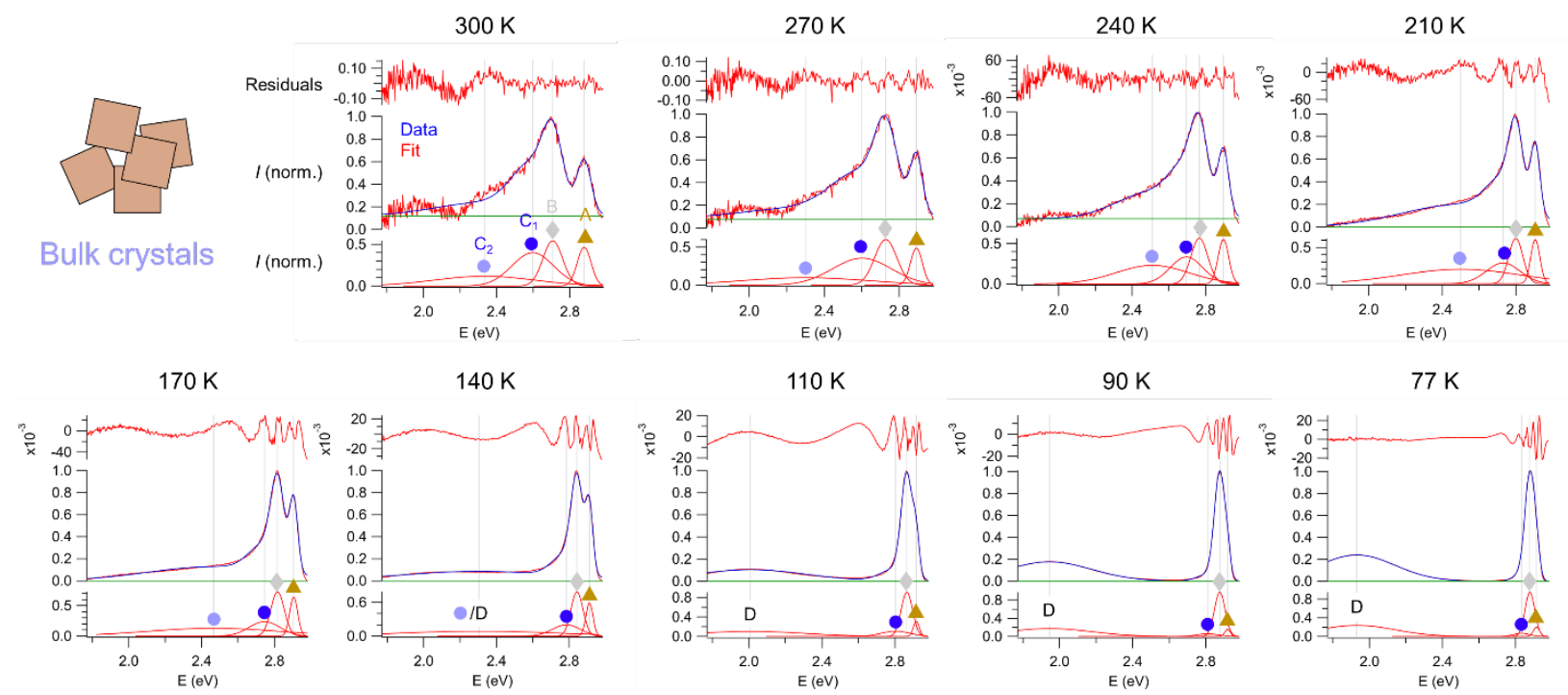

Figure S11. Gaussian fitting of the bulk crystals with cooling, assuming that $C$ is two Gaussians, $C_{1}$ and $C_{2}$, at all temperatures.

We can plot the energy shift and Gaussian area of $\mathrm{C}_{2}$. No discernable trends are seen until $110 \mathrm{~K}$, at which point the behavior of the STE peak D is obvious (Figure S12):

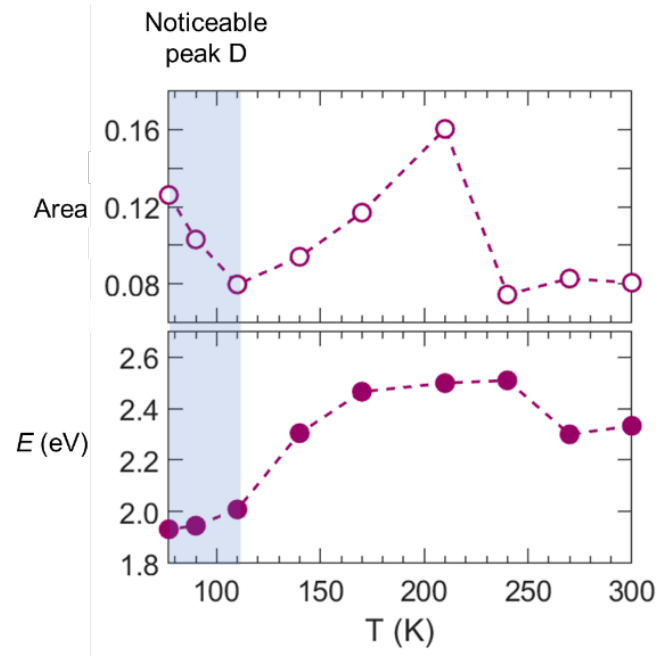

Figure S12. Evolution of the fourth Gaussian from Figure S11. This Gaussian becomes peak D at low temperatures (blue shading). See also Figure S15 for comparison with film behavior. 
Since it is not clear how many Gaussians should be used to fit $C$, we can also look at the residuals, once peaks A, B and D are accounted for:
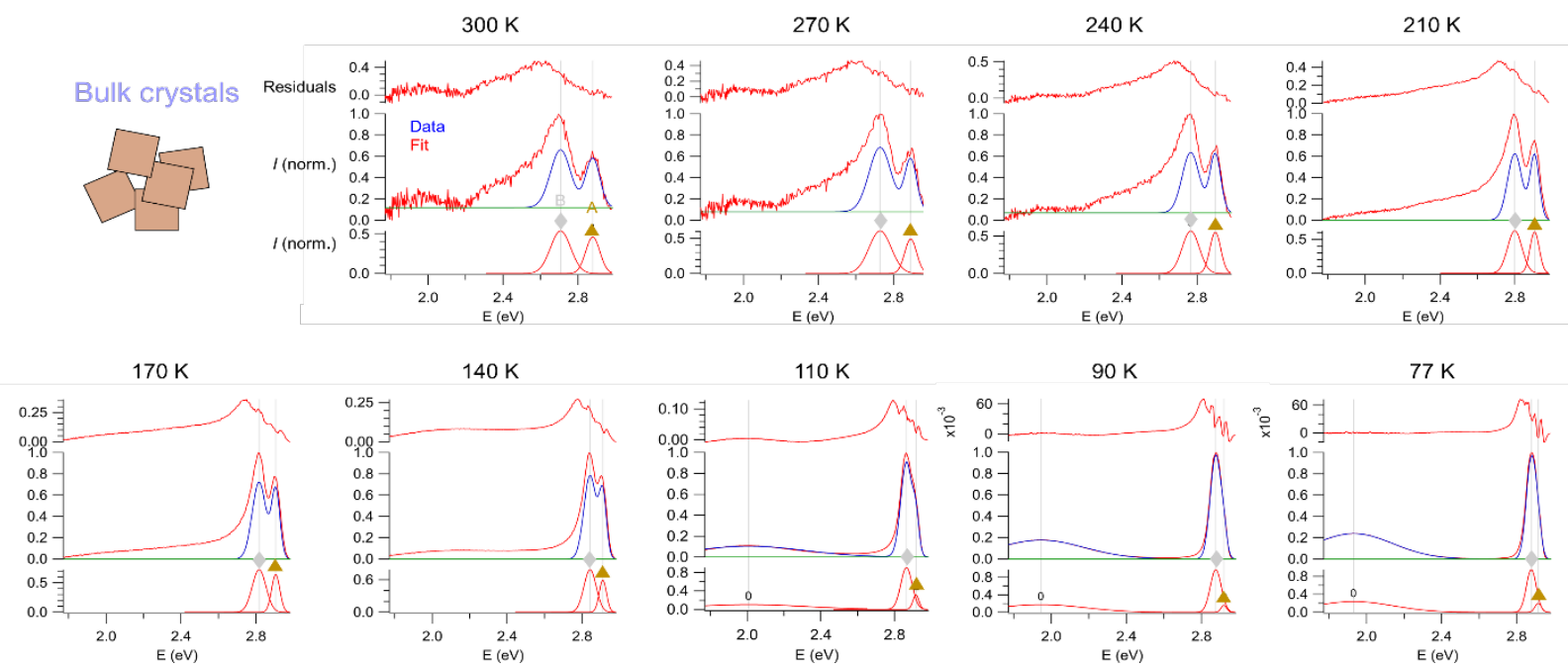

Figure S13. Gaussian fitting (bulk crystals) of A, B, and D, showing the residuals (assumed to be peak C), if the Gaussians $\mathrm{C}_{1}$ and $\mathrm{C}_{2}$ are deleted from Figure S11.

From Figure S13, it is clear that a broad peak is present at room temperature. This peak is roughly symmetric at $300 \mathrm{~K}$, but becomes gradually weaker and less symmetric cooler temperatures, suggesting that $\mathrm{C}$ is both decreasing in intensity and blue-shifting. The asymmetry also suggests overlap between C and A/B, as also suggested by Figures S10 and S11.

Finally, if we assume no overlap between C and A/B (Figure S14), we see from the residuals that C seems to strongly blue-shift.

\section{Conclusion: $\mathrm{C}$ blue-shifts and decreases in intensity with cooling.}
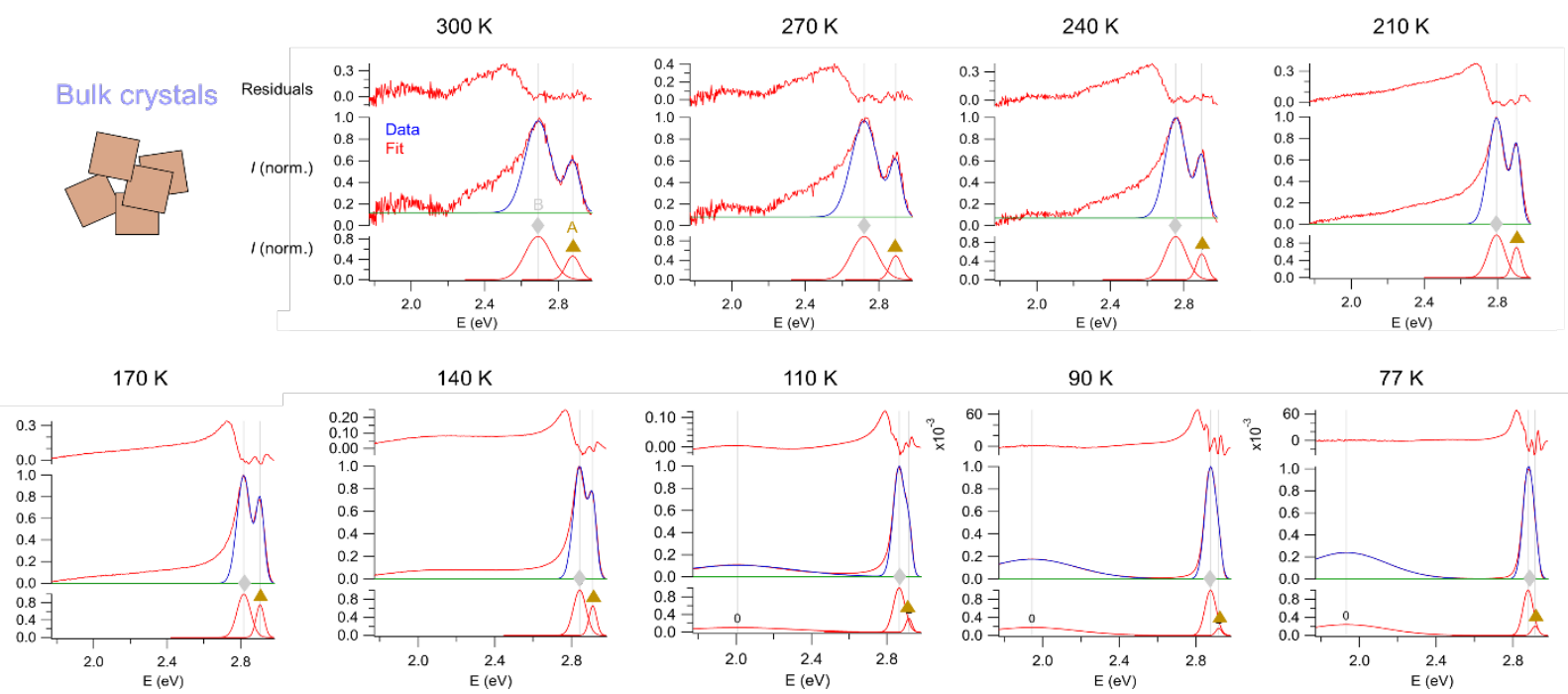

Figure S14. Gaussian fitting (bulk crystals) of A, B, and D, showing the residuals (assumed to be peak C), if there is no overlap between A, B and C (unlikely). 
(a)

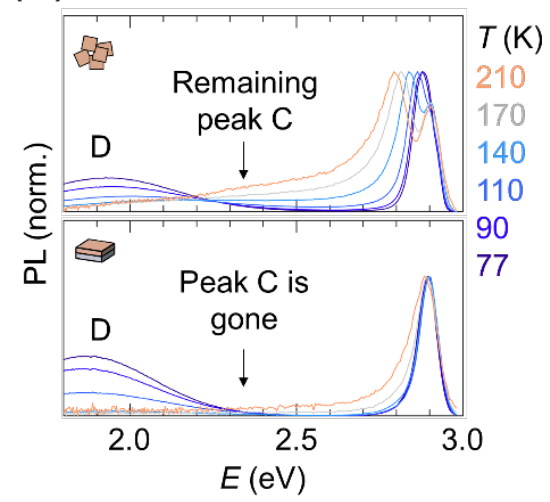

(b)

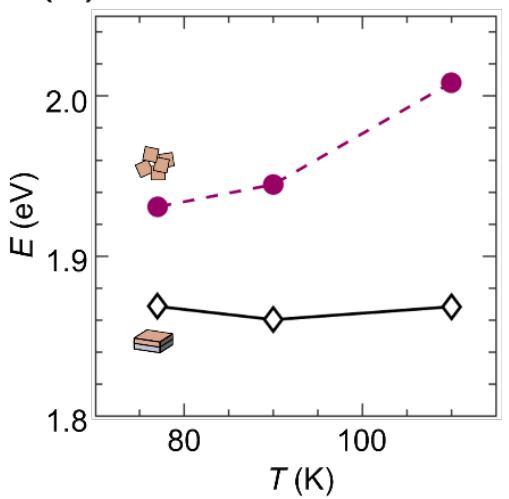

(c)

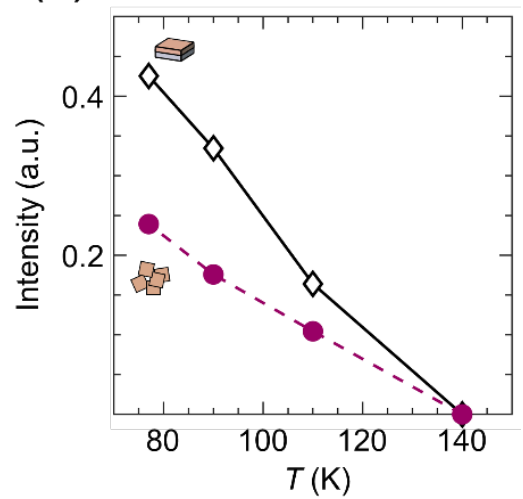

Figure S15. Temperature-dependent photoluminescence emission of the bulk crystals and film of (EA) ${ }_{4} \mathrm{~Pb}_{3} \mathrm{Br}_{10}$ (see Figure 4), focusing on the peak at $1.9 \mathrm{eV}$ (peak D). (a) Spectra for $210 \mathrm{~K}$ and below, (b) Changes to peak position and (c) Changes to peak intensity, obtained from Gaussian fits (intensity was used rather than area, as the peaks are cut off).

Peak D gradually increases in intensity below $140 \mathrm{~K}$ for both bulk crystals and films. This behavior is consistent with that of self-trapped excitons studied elsewhere. 15,16 The red-shift with cooling (bulk crystals) is further consistent with STEs that are associated with localized polarons. ${ }^{16}$ However, D behaves different in bulk crystals vs. films: the films exhibit more intense peak $\mathrm{D}$, and the bulk crystals exhibit a more intense red-shift. While peak D is different in nature than B and C, here again, Peak D (STE) behaves differently in bulk crystals and in films. 


\section{O Bulk crystal \\ $\diamond$ Film}

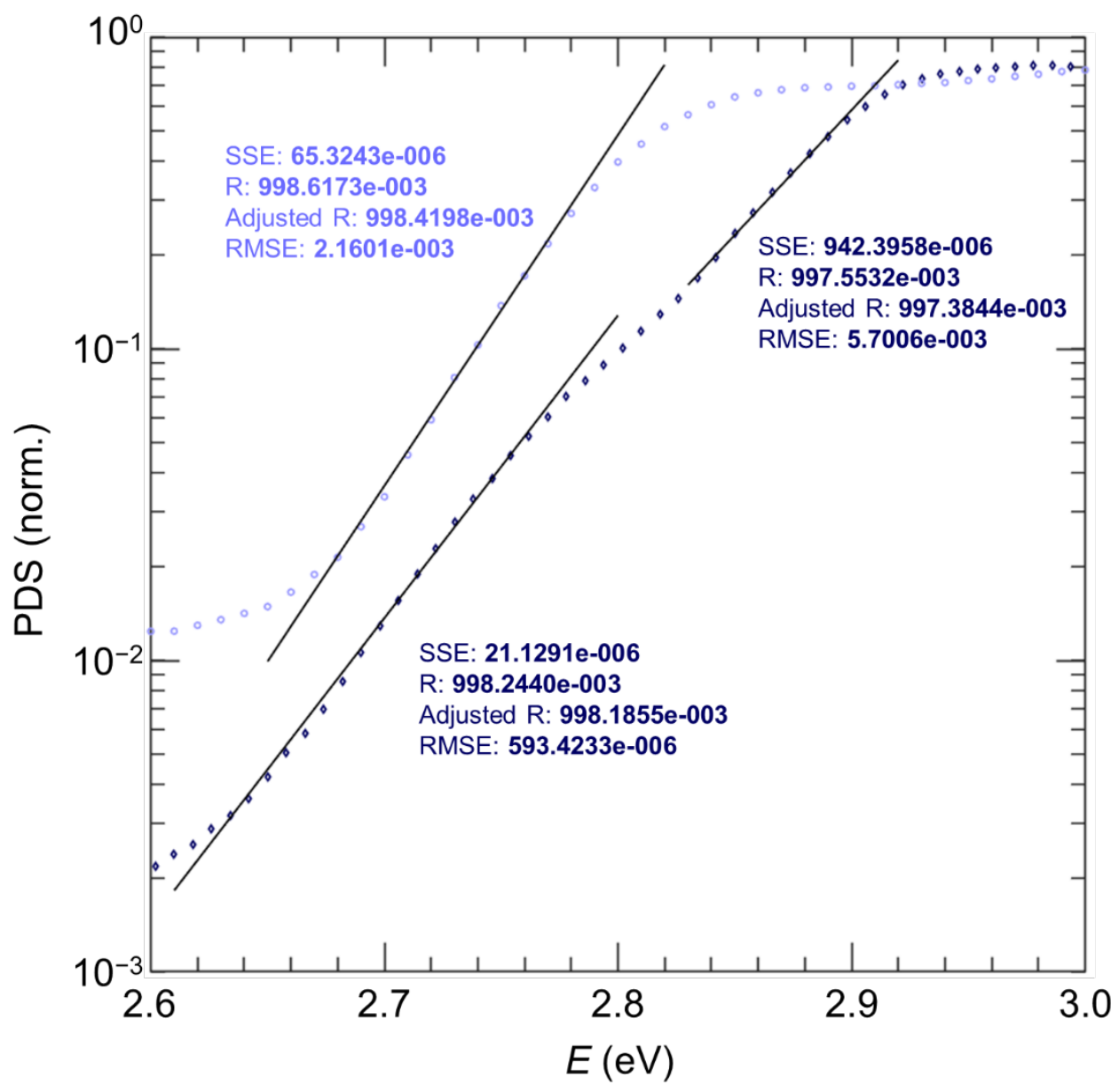

Figure S16. PDS in the 2.6-3.0 eV region for the bulk crystal and film, with goodness of fit parameters from MATLAB: the sum of squares due to error (SSE), R-square (R), Adjusted R-square, and the Root mean squared error (RMSE). 

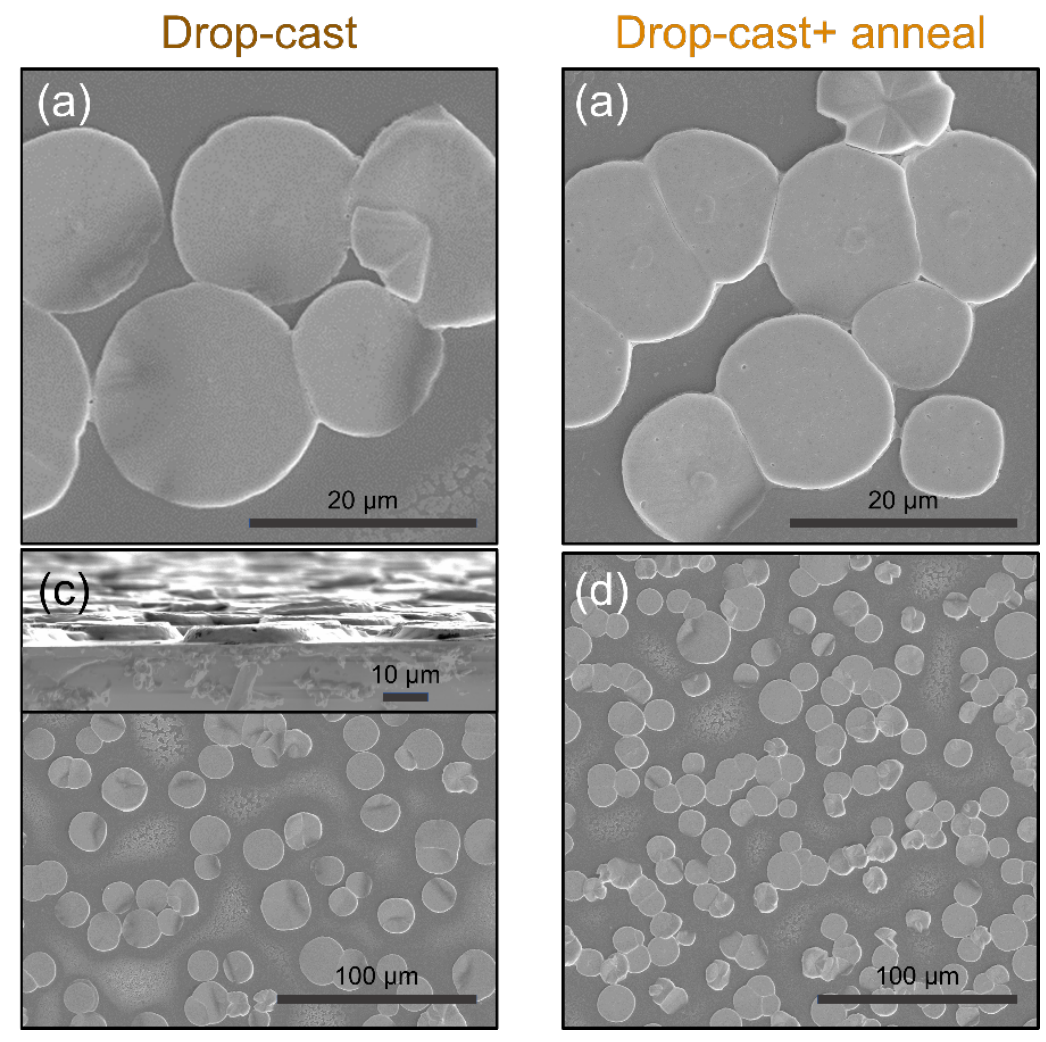

Figure S17. SEM of the drop-cast films from Figure 6, with the inset of (c) showing a cross-section. 

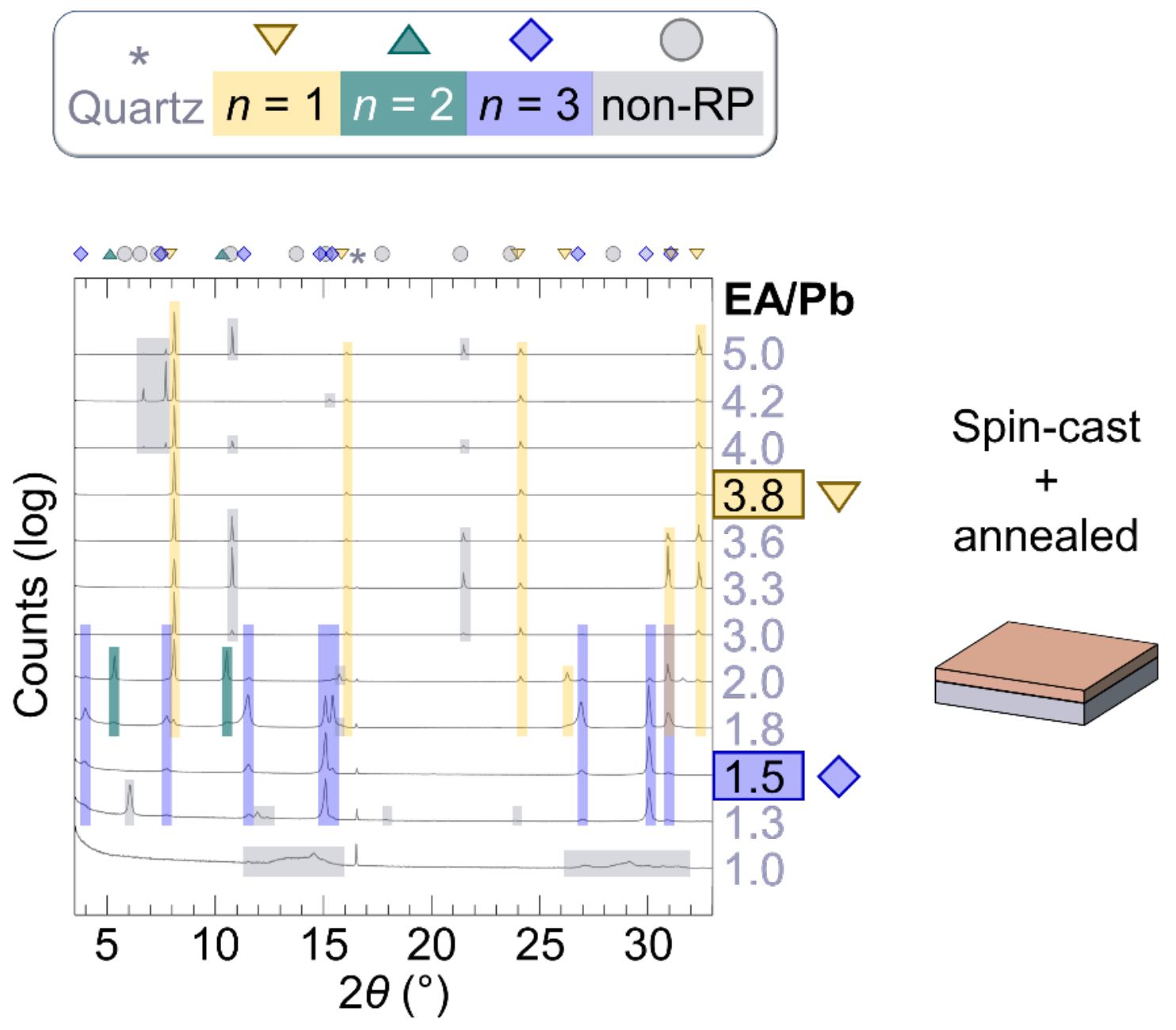

Figure S18. XRD patterns of spin-cast and annealed films, for which the EA/Pb molar ratio of the precursor solutions was steadily increased. Only for $\mathrm{EA} / \mathrm{Pb}=1.5$ and for $\mathrm{EA} / \mathrm{Pb}=3.8$ were phase pure films obtained, and these were $n=3$ and $n=1$ films respectively. However, the large excess of EA (nearly twice the stoichiometric amount) needed for the $n=1$ film formation shows that $n=1$ is not easily formed. No 3D film or phase was formed.

To further understand the limits of phase formation in the (EA) $2(E A)_{n-1} \mathrm{~Pb}_{n} B r_{3 n+1}$ family, we tried to make ethylammonium-based RP films of different $n$ via spin-casting (Figure S18). The molar ratio of $\mathrm{EABr}: \mathrm{PbBr}_{2}\left(\mathrm{EA} / \mathrm{Pb}\right.$ for short) in the precursor solutions was steadily increased from $1.0\left(\mathrm{EAPbBr}_{3}\right.$ $3 \mathrm{D}$ stoichiometry) to 3.8 (nearly twice the $n=1(\mathrm{EA})_{2} \mathrm{PbBr}_{4}$ stoichiometry). For $\mathrm{EA} / \mathrm{Pb}=1.0$, which corresponds stoichiometrically to $\mathrm{EAPbB}_{3}$, we obtained broad peaks, likely indicating an amorphous phase (the film was also non-emissive). A slight excess of EA is thought to be needed for $n=3$ film formation because $\mathrm{Pb}$ and $\mathrm{Br}$ form strong complexes with DMF. ${ }^{17}$ In addition, no 3D-like phase was conclusively observed in any of the EA compositions tested. Despite the large range of EA/Pb molar ratios tested, only the $n=1$ and $n=3$ compositions could be cast as phase-pure films. 

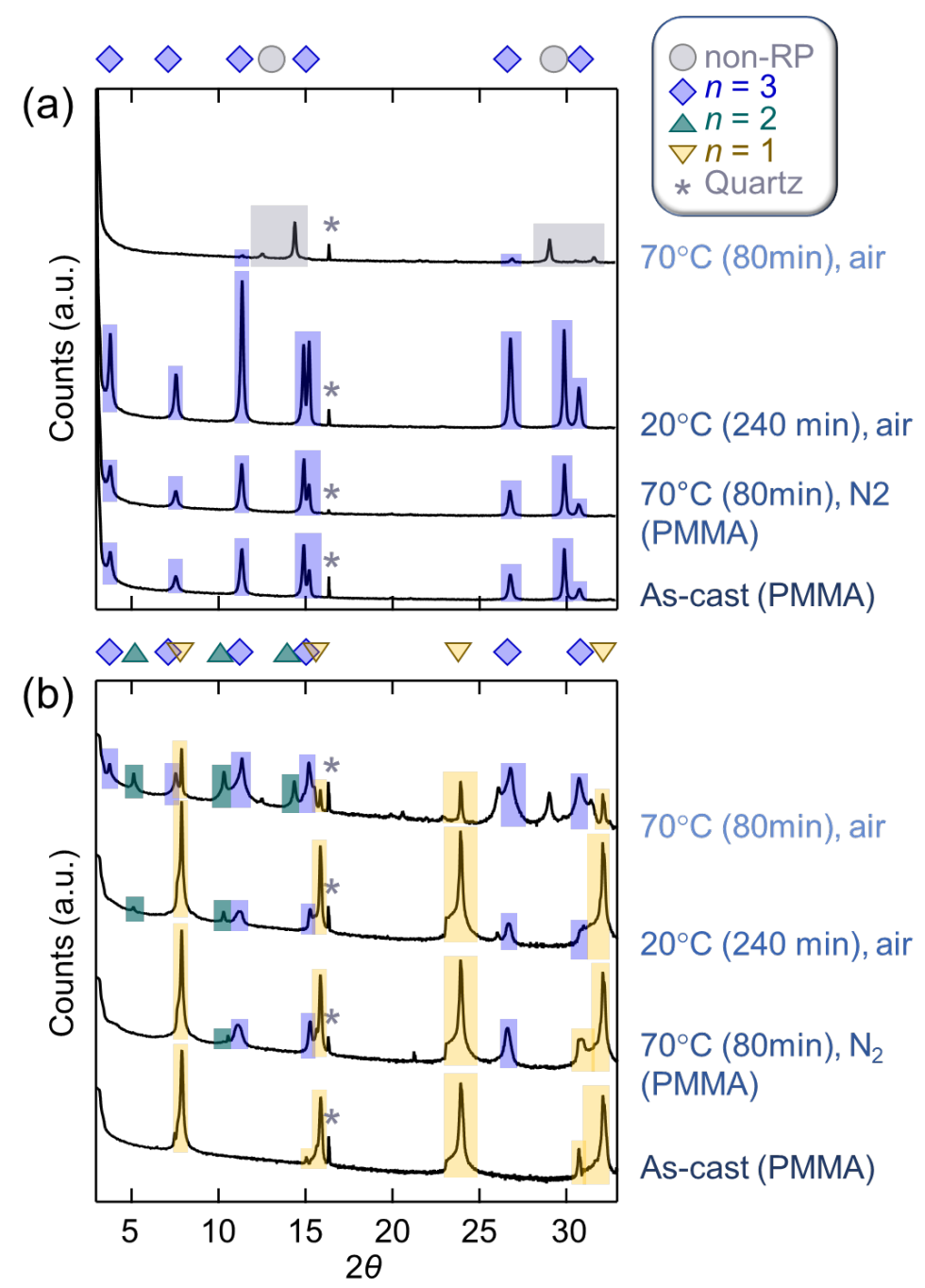

Figure S19. XRD patterns under different stability testing conditions for (a) $n=3$ and (b) $n=1$ spincast films.

We examined the stability of $n=1$ and $n=3$ spin-cast films, in order to better understand which phases are thermodynamically favorable (Figure S19), and summarized the results in Figure 7. Films were spin-cast with or without the PMMA capping layer. Four testing conditions were employed, to test stability to heat and air: (1) control: as-cast (with PMMA capping), (2) heat test: heating at $70^{\circ} \mathrm{C}$ in the glove box (nitrogen environment) for $80 \mathrm{~min}$ (with PMMA capping), (3) air test: storage in air at $20^{\circ} \mathrm{C}$ for $240 \mathrm{~min}$ (no PMMA capping) and (4) heat and air test: heating at $70^{\circ} \mathrm{C}$ in air for 80 min (no PMMA capping).

The $n=3$ phase proved more thermodynamically stable than the $n=1$ (Figure S19). Heating encapsulated $n=3$ films in a nitrogen environment produced no change in structure, indicating that $n=3$ is thermodynamically stable under mild conditions. In contrast, this mild heating resulted in clear phase-change in the encapsulated $n=1$. The novel peaks were tentatively assigned to $n=3$ and $n=2$, although the low number of non- $n=1$ peaks made full assignment challenging. The greater thermodynamic stability of the $n=3$ may help explain why it formed more favorably in films. 
The $n=3$ also appeared to be much more air-stable than the $n=1$ (Figure S19). Short, unencapsulated storage in air resulted in retention of the $n=3$ phase, albeit with large peak intensity increase and out-of-plane (0k0) stacking preference. Humidity-induced increase in grain size has been previously reported for $\mathrm{MAPbI}_{3} ;{ }^{18}$ it is possible that humidity caused recrystallization here as well. This rearrangement is encouraging, as it suggests that post-casting treatments could control film orientation for $n=3$. In contrast, short, unencapsulated storage in air resulted in degradation of the $n=1$ film. Finally, heating unencapsulated $n=3$ and $n=1$ films in air resulted in obvious degradation of both films, indicating that encapsulation will likely be necessary for ethylammonium RP device use. The originally- $n=3$ film contained peaks near 14 and 29 degrees, perhaps indicating a 1D degradation product. Interestingly, the originally- $n=1$ film now very clearly contained $n=3$ as well as some $n=2$, indicating that $n=3$ is a degradation product $n=1$. The $n=1$ to $n=3$ phase change in heated unencapsulated films also suggests that ethylammonium desorbed. Overall, the $n=3$ EA RP was considerably more stable than the $n=1$. 


\section{Further Discussion on 2D and 3D phases with the EA cation}

Previous work that attempted to make films of 3D $\mathrm{EAPbBr}_{3}$ found that a 2D phase formed instead. ${ }^{19}$ Given the location of the XRD peaks of this film, it is likely that the $n=3$ phase formed in the film in the prior study. ${ }^{19}$ Perhaps the use of an antisolvent in prior work helped $n=3$ phase formation, which might be otherwise hindered by excess of lead in the $\mathrm{EAPbBr}_{3}$ solution stoichiometry. Another report claimed formation of $\mathrm{EAPbBr}_{3}$ in nanocrystals. ${ }^{20}$ However, the optical properties of the resulting nanocrystals matched $(\mathrm{EA})_{4} \mathrm{~Pb}_{3} \mathrm{Br}_{10}$, and XRD data was not collected in the range characteristic for 2D phases (below $2 \theta=10^{\circ}$ ), ${ }^{20}$ suggesting that the nanocrystals might instead be $(\mathrm{EA})_{4} \mathrm{~Pb}_{3} \mathrm{Br}_{10}$. EA can be incorporated into 3D phases, provided there is a small A-site cation (e.g. MA) that it can alloy with, to form (EA,MA) $\mathrm{PbBr}_{3} .{ }^{19}$ 


\section{References}

(1) Kieslich, G.; Sun, S.; Cheetham, A. K. Solid-State Principles Applied to OrganicInorganic Perovskites: New Tricks for an Old Dog. Chem. Sci. 2014, 5 (12), 4712-4715.

(2) Becker, M.; Klüner, T.; Wark, M. Formation of Hybrid ABX 3 Perovskite Compounds for Solar Cell Application: First-Principles Calculations of Effective Ionic Radii and Determination of Tolerance Factors. Dalt. Trans. 2017, 46 (11), 3500-3509.

(3) Goldschmidt, V. V. Die Gesetze Der Krystallochemie. Naturwissenschaften 1926, 14, 477-485.

(4) Dow, J. D.; Redfield, D. Toward a Unified Theory of Urbach's Rule and Exponential Absorption Edges. Phys. Rev. B 1972, 5 (2), 594-610.

(5) Dahlman, C. J.; Decrescent, R. A.; Venkatesan, N. R.; Kennard, R. M.; Wu, G.; Everest, M. A.; Schuller, J. A.; Chabinyc, M. L. Controlling Solvate Intermediate Growth for PhasePure Organic Lead Iodide Ruddlesden-Popper $\left(\mathrm{C}_{4} \mathrm{H}_{9} \mathrm{NH}_{3}\right)_{2}\left(\mathrm{CH}_{3} \mathrm{NH}_{3}\right)_{n-1} \mathrm{~Pb}_{n} \mathrm{I}_{3 n+1}$ Perovskite Thin Films. Chem. Mater. 2019, 31 (15), 5832-5844.

(6) Ma, Z.; Li, F.; Sui, L.; Shi, Y.; Fu, R.; Yuan, K.; Xiao, G.; Zou, B. Tunable Color Temperatures and Emission Enhancement in 1D Halide Perovskites under High Pressure. Adv. Opt. Mater. 2020, 8 (18), 1-7.

(7) Tu, Q.; Spanopoulos, I.; Hao, S.; Wolverton, C.; Kanatzidis, M. G.; Shekhawat, G. S.; Dravid, V. P. Out-of-Plane Mechanical Properties of 2D Hybrid Organic-Inorganic Perovskites by Nanoindentation. ACS Appl. Mater. Interfaces 2018, 10 (26), 2216722173.

(8) Feng, J. Mechanical Properties of Hybrid Organic-Inorganic $\mathrm{CH}_{3} \mathrm{NH}_{3} \mathrm{BX}_{3}(\mathrm{~B}=\mathrm{Sn}, \mathrm{Pb} ; \mathrm{X}$ $=\mathrm{Br}$, I) Perovskites for Solar Cell Absorbers. APL Mater. 2014, 2 (8), 081801.

(9) Luo, B.; Guo, Y.; Li, X.; Xiao, Y.; Huang, X.; Zhang, J. Z. Efficient Trap-Mediated $\mathrm{Mn}^{2+}$ Dopant Emission in Two Dimensional Single-Layered Perovskite $\left(\mathrm{CH}_{3} \mathrm{CH}_{2} \mathrm{NH}_{3}\right)_{2} \mathrm{PbBr}_{4}$. J. Phys. Chem. C 2019, 123 (23), 14239-14245.

(10) Mao, L.; Wu, Y.; Stoumpos, C. C.; Traore, B.; Katan, C.; Even, J.; Wasielewski, M. R.; Kanatzidis, M. G. Tunable White-Light Emission in Single-Cation-Templated ThreeLayered 2D Perovskites $\left(\mathrm{CH}_{3} \mathrm{CH}_{2} \mathrm{NH}_{3}\right)_{4} \mathrm{~Pb}_{3} \mathrm{Br}_{10-x} \mathrm{Cl} x_{x}$ J. Am. Chem. Soc. 2017, 139 (34), 11956-11963.

(11) Venkatesan, N. R.; Kennard, R. M.; DeCrescent, R. A.; Nakayama, H.; Dahlman, C. J.; Perry, E. E.; Schuller, J.; Chabinyc, M. L. Phase Intergrowth and Structural Defects in Organic Metal Halide Ruddlesden-Popper Thin Films. Chem. Mater. 2018, 30, 86158623.

(12) Decrescent, R. A.; Du, X.; Kennard, R. M.; Venkatesan, N. R.; Dahlman, C. J.; Chabinyc, M. L.; Schuller, J. A. Even-Parity Self-Trapped Excitons Lead to Magnetic Dipole Radiation in Two-Dimensional Lead Halide Perovskites. ACS Nano 2020, 14 (7), 8958-8968.

(13) DeCrescent, R. A.; Venkatesan, N. R.; Dahlman, C. J.; Kennard, R. M.; Zhang, X.; Li, W.; $\mathrm{Du}, \mathrm{X}$;; Chabinyc, M. L.; Zia, R.; Schuller, J. A. Bright Magnetic Dipole Radiation from Two-Dimensional Lead-Halide Perovskites. Sci. Adv. 2020, No. February, 1-11.

(14) Mooney, J.; Kambhampati, P. Get the Basics Right: Jacobian Conversion of Wavelength and Energy Scales for Quantitative Analysis of Emission Spectra. J. Phys. Chem. Lett. 2013, 4 (19), 3316-3318.

(15) Hu, T.; Smith, M. D.; Dohner, E. R.; Sher, M. J.; Wu, X.; Trinh, M. T.; Fisher, A.; Corbett, J.; 
Zhu, X. Y.; Karunadasa, H. I.; Lindenberg, A.M. Mechanism for Broadband White-Light Emission from Two-Dimensional (110) Hybrid Perovskites. J. Phys. Chem. Lett. 2016, 7 (12), 2258-2263.

(16) Cortecchia, D.; Yin, J.; Bruno, A.; Lo, S. Z. A.; Gurzadyan, G. G.; Mhaisalkar, S.; Brédas, J. L.; Soci, C. Polaron Self-Localization in White-Light Emitting Hybrid Perovskites. J. Mater. Chem. C 2017, 5 (11), 2771-2780.

(17) Yoon, S. J.; Stamplecoskie, K. G.; Kamat, P. V. How Lead Halide Complex Chemistry Dictates the Composition of Mixed Halide Perovskites. J. Phys. Chem. Lett. 2016, 7 (7), 1368-1373.

(18) Chiang, C. H.; Wu, C. G. A Method for the Preparation of Highly Oriented $\mathrm{MAPbI}_{3}$ Crystallites for High-Efficiency Perovskite Solar Cells to Achieve an 86\% Fill Factor. ACS Nano 2018, 12(10), 10355-10364.

(19) Gholipour, S.; Ali, A. M.; Correa-Baena, J. P.; Turren-Cruz, S. H.; Tajabadi, F.; Tress, W.; Taghavinia, N.; Grätzel, M.; Abate, A.; De Angelis, F.; Gaggioli, C. A. ; Mosconi, E. ; Hagfeldt, A. ; Saliba, M. Globularity-Selected Large Molecules for a New Generation of Multication Perovskites. Adv. Mater. 2017, 29 (38), 1-9.

(20) Mittal, M.; Jana, A.; Sarkar, S.; Mahadevan, P.; Sapra, S. Size of the Organic Cation Tunes the Band Gap of Colloidal Organolead Bromide Perovskite Nanocrystals. J. Phys. Chem. Lett. 2016, 7 (16), 3270-3277. 